\title{
COSMIC RAY RESULTS ON THE $A$ DEPENDENCE OF MULTIPLICITY AND ANGULAR DISTRIBUTIONS IN PROTON NUCLEAR INTERACTIONS ABOVE $100 \mathrm{GeV}^{\text {s }}$
}

\author{
P.R. VISHWANATH, A.E. BUSSIAN ${ }^{* 1}$, L.W. JONES and D.E. LYON, Jr. ${ }^{* 2}$ \\ University of Michigan, Ann Arbor, Michigan 48104, USA \\ and \\ J.G. LEARNED, D.D. REEDER and R.J. WILKES ${ }^{*}$ \\ University of Wisconsin, Madison, Wisconsin 53706, USA
}

Received 20 November 1974

\begin{abstract}
A calorimeter-spark chamber system was used to collect data on several hundred proton-nucleus interactions above $100 \mathrm{GeV}$ using targets of $\mathrm{C}, \mathrm{Al}, \mathrm{Fe}, \mathrm{Sn}$ and $\mathrm{Pb}$. The average charged prong multiplicity is found to depend on atomic mass number as $\left\langle n_{\mathrm{c}}\right\rangle=\left\langle n_{\mathrm{c}}\right\rangle(\mathrm{p}-\mathrm{p}) A^{x}$ where $x=0.129 \pm 0.004$, with the dominant increase in multiplicity occurring in the backward (p-p c.m.) hemisphere. The value of $x$ shows no significant energy dependence.
\end{abstract}

A cosmic ray experiment is reported on interactions of hadrons (mostly protons) of $E>100 \mathrm{GeV}$ with nuclei of $\mathrm{C}$. $\mathrm{Al}, \mathrm{Fe}, \mathrm{Sn}$ and $\mathrm{Pb}[1]$. Data were collected on charged prong multiplicities and angular distributions from a total of 500 nuclear interactions in the four metallic targets. Two separate sets of data of the same sort were taken with a carbon target $[2,3]$; the definitive set included 355 target interactions. The nucleus may be considered to be a microscopic laboratory for investigations of the space-time development of the final state of a proton-nucleon interaction [410]. Thus, exploration of the variation of inclusive parameters of proton-nuclear collisions as functions of nuclear mass number and energy might elucidate various theoretical models of the nucleon-nucleon interaction. In addition, a systematic phenomenological understanding of these parameters may be of value in interpreting other high energy phenomena involving nuclear targets, such as extensive air showers and data on high energy muon or neutrino interactions [1113].

\footnotetext{
Supported by the U.S. National Science Foundation.

*1 Division of Science and Mathematics, Community College of Denver, Red Rocks Campus, Lakewood, Colorado 80215, USA.

*2 Stanford Linear Accelerator Center, Stanford, California 94305, USA.

*3 CERN, 1211 Geneva 23, Switzerland
}

The experiment employed the apparatus described previously at Echo Lake, Colorado at an elevation of $3230 \mathrm{~m} \mathrm{[14]}$. The detector included wide gap spark chambers of $4 \mathrm{~m}^{2}$ area above and below the target, an ionization calorimeter of $1170 \mathrm{~g} \mathrm{~cm}^{-2}$ of iron, and an array of air-shower anticoincidence counters. The apparatus, which had an admittance (geometrical factor) of $0.94 \mathrm{~m}^{2} \mathrm{sr}$, is depicted schematically in fig. 1 . Each metallic element studied was in the form of 6 plates, each $106 \times 212 \mathrm{~cm}^{2}$, stacked vertically with about $15 \mathrm{~cm}$ vertical spacing. The carbon target was in the form of a single rectangular layer $203 \times 183 \mathrm{~cm}^{2}$.

The final data sample contains only events with hadronic interactions in the target which exceeded a threshold energy cut and satisfied fiducial boundary conditions. For each event the space angles of the incident particle, the vertex coordinates of the interaction, and the projected angles of the reaction products with respect to the incident particle were obtained ${ }^{\ddagger 1}$. Several systematic biases required correction in the data analysis. These included four classes of effects.

\#1 The correlation of tracks in the two $90^{\circ}$ stereoscopic views was not possible so that the angles obtained were those of the tracks projected onto a plane containing the incident particle and a normal to the optic axis of the camera. 


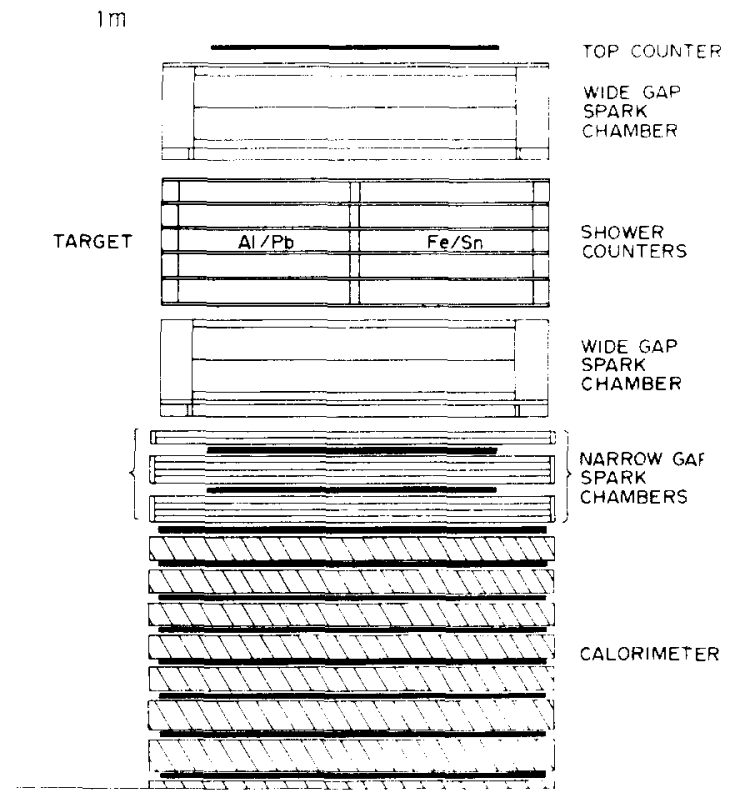

Fig. 1. Vertical section of the experimental configuration. (Shower counters are around the target and are not shown explicitly in the diagram). The absorber material in the calorimeter is iron.

(I) Overestimation of multiplicity of tracks due to secondary interactions, $\gamma$-ray conversions, and $\delta$ ray production below the event vertex.

(II) Underestimation of multiplicity due to stopping of low-energy particles in the target and to spark chamber inefficiences. The observed tracks did not generally include nuclear fragments because of the target thickness. Tracks separated by less than $2 \mathrm{mr}$ were not resolved, and some wide-angle tracks escaped the sensitive volume of the spark chambers. A more serious bias was the spark chamber inefficiencies for larger-angle tracks and high multiplicity events. A major effort was made to systematically calibrate and evaluate these inefficiences and to correct for them $\mathrm{m}^{\neq 2}$.

\$2 These chamber inefficiencies account for the discrepancy between the published Echo Lake hy drogen data and the subsequent FNAL bubble chamber results on average charged multiplicities. Subsequent reanalysis including results of this inefficiency evaluation has greatly improved the agreement between the two sets of hydrogen data, as reported in refs. [1] and [12] and in University of Michigan report UM HE 73-21.
(III) Underestimation of energy by the calorimeter. Besides a systematic shift in energy calibration resulting from unsampled energy losses, the energy of some events was sampled by the calorimeter less efficiently than others, due to escape of large angle prongs from the fiducial volume of the calorimeter. This resulted in a steep decrease in number of events with increasing vertical distance of the event vertex from the calorimeter and increasing atomic weight of the target.

(IV) Beam contamination: From earlier studies, it is known that about $30 \%$ of the incident charged hadrons which trigger the system are pions. No correction has been made for this effect, and the analysis was carried through assuming only incident protons.

In order to correct for these systematic biases, two somewhat arbitrary but reasonable Monte Carlo models were constructed for p-nucleus interactions and events were generated with them from each of the target elements. The known experimental biases were then applied to these events and the resulting distributions compared with the experimentally observed events. Finally the observed distributions were modified by the inverse of the effects of the experimental biases thus determined. The Monte Carlo models assumed a proton-nucleon interaction in the target nucleus with a final state given by the observed properties of $p-p$ collisions. The on-going beam nucleon was then allowed to interact within the nucleus one or more times, while the other produced particles were assumed to escape the nucleus without subsequent interactions. The two models differed only in the maximum number of intranuclear collisions allowed for the beam nucleon. This procedure of corrections to the biased data based on an empirical Monte Carlo model is dangerous, as false aspects of the model might bias the corrections to the data and still produce superficial agreement with observables. A measure of the uncertainty introduced by the Monte Carlo corrections is gained in comparing the raw data on average charged multiplicity with the corrected values. The errors indicated on the graphs and talbes are statistical only and do not reflect these systematic uncertainties.

The results on average charged multiplicity from table 1 are given in fig. $2 \mathrm{a}$ in terms of the parameter $R$ as a function of atomic weight $A$, where $R(A)=$ $\left\langle n_{\mathrm{c}}\right\rangle(\mathrm{p}-\mathrm{A}) /\left\langle n_{\mathrm{c}}\right\rangle(\mathrm{p}-\mathrm{p})$, and the quoted energy is an average for each contiguous bin. The value of $\left\langle n_{c}\right\rangle(p-p)$ is 
Table 1

Observed and corrected average charged prong multiplicities

\begin{tabular}{|c|c|c|c|c|c|c|c|c|}
\hline $\begin{array}{l}\text { Target } \\
\text { element }\end{array}$ & $\begin{array}{l}\text { Thickness } \\
\text { of the } \\
\text { total target } \\
\text { in } \mathrm{g} / \mathrm{cm}^{2}\end{array}$ & $\begin{array}{l}\text { Average } \\
\text { energy } \\
\text { in } \mathrm{GeV}^{(a)}\end{array}$ & $\begin{array}{l}\text { No. of } \\
\text { inter- } \\
\text { actions }\end{array}$ & $\begin{array}{l}\text { Average } \\
\text { charged } \\
\text { multiplicity } \\
\text { (raw data) }\end{array}$ & $\begin{array}{l}\text { Monte Carlo } \\
\text { corrections } \\
\text { Model A }\end{array}$ & $\begin{array}{l}\text { (b) } \\
\text { Model B }\end{array}$ & $\begin{array}{l}\text { Corrected } \\
\text { average } \\
\text { charged } \\
\text { multiplicity }\end{array}$ & $R^{(\mathrm{c})}$ \\
\hline C & 17.54 & $\begin{array}{r}85 \\
120 \\
173 \\
379\end{array}$ & $\begin{array}{r}121 \\
105 \\
68 \\
61\end{array}$ & $\begin{array}{l}6.04 \pm 0.28 \\
7.80 \pm 0.35 \\
8.24 \pm 0.54 \\
7.63 \pm 0.43\end{array}$ & $\begin{array}{l}1.30 \\
1.30 \\
1.39 \\
1.61\end{array}$ & & $\begin{array}{r}7.89 \pm 0.34 \\
10.13 \pm 0.42 \\
11.44 \pm 0.64 \\
12.28 \pm 0.83\end{array}$ & $\begin{array}{l}1.26 \pm 0.08 \\
1.48 \pm 0.09 \\
1.55 \pm 0.10 \\
1.40 \pm 0.11\end{array}$ \\
\hline $\mathrm{Al}$ & 20.67 & $\begin{array}{l}160 \\
260 \\
520\end{array}$ & $\begin{array}{l}70 \\
67 \\
24\end{array}$ & $\begin{array}{l}7.67 \pm 0.42 \\
8.55 \pm 0.51 \\
8.08 \pm 1.05\end{array}$ & $\begin{array}{l}1.47 \\
1.48 \\
1.67\end{array}$ & $\begin{array}{l}1.46 \\
1.53 \\
1.80\end{array}$ & $\begin{array}{l}11.25 \pm 0.76 \\
12.87 \pm 1.18 \\
14.02 \pm 2.49\end{array}$ & $\begin{array}{l}1.54 \pm 0.10 \\
1.58 \pm 0.10 \\
1.50 \pm 0.26\end{array}$ \\
\hline $\mathrm{Fe}$ & 29.98 & $\begin{array}{l}160 \\
260 \\
520\end{array}$ & $\begin{array}{l}60 \\
68 \\
26\end{array}$ & $\begin{array}{r}8.66 \pm 0.53 \\
10.28 \pm 0.61 \\
9.96 \pm 1.05\end{array}$ & $\begin{array}{l}1.41 \\
1.29 \\
1.59\end{array}$ & $\begin{array}{l}1.42 \\
1.28 \\
1.64\end{array}$ & $\begin{array}{l}12.25 \pm 0.89 \\
13.21 \pm 1.16 \\
16.05 \pm 2.90\end{array}$ & $\begin{array}{l}1.68 \pm 0.12 \\
1.62 \pm 0.12 \\
1.72 \pm 0.31\end{array}$ \\
\hline $\mathrm{Sn}$ & 27.85 & $\begin{array}{l}160 \\
260 \\
520\end{array}$ & $\begin{array}{l}49 \\
51 \\
11\end{array}$ & $\begin{array}{r}9.77 \pm 0.73 \\
10.72 \pm 0.75 \\
9.09 \pm 1.56\end{array}$ & $\begin{array}{l}1.39 \\
1.41 \\
1.96\end{array}$ & $\begin{array}{l}1.33 \\
1.41 \\
1.79\end{array}$ & $\begin{array}{l}13.31 \pm 1.13 \\
15.14 \pm 1.40 \\
17.05 \pm 3.60\end{array}$ & $\begin{array}{l}1.82 \pm 0.15 \\
1.86 \pm 0.15 \\
1.82 \pm 0.35\end{array}$ \\
\hline $\mathrm{Pb}$ & 32.43 & $\begin{array}{l}160 \\
260\end{array}$ & $\begin{array}{l}40 \\
39\end{array}$ & $\begin{array}{l}10.42 \pm 0.75 \\
10.54 \pm 0.77\end{array}$ & $\begin{array}{l}1.42 \\
1.51\end{array}$ & $\begin{array}{l}1.41 \\
1.45\end{array}$ & $\begin{array}{l}14.75 \pm 1.41 \\
15.61 \pm 1.51\end{array}$ & $\begin{array}{l}2.02 \pm 0.20 \\
1.92 \pm 0.18\end{array}$ \\
\hline
\end{tabular}

a) The energy bins are contiguous and are $\pm 16 \%$ wide for the $\mathrm{C}$ data and $\pm 33 \%$ wide for the metal data.

b) The data fell between the predictions of model $\mathrm{A}$ and model $\mathrm{B}$, so that an average was used to generate the corrected $\left\langle n_{\mathrm{C}}\right\rangle$. The corrections, while being functions of the energy and the observed vertex distributions, are quite model independent. Only one Monte Carlo model was used with the carbon data, differing in detail from models $\mathbf{A}$ and $\mathbf{B}$.

c) $R=\left\langle n_{\mathrm{c}}\right\rangle(\mathrm{p}-\mathrm{A}) /\left\langle n_{\mathrm{c}}\right\rangle(\mathrm{p}-\mathrm{p})$, where $\left\langle n_{\mathrm{c}}\right\rangle(\mathrm{p}-\mathrm{p})$ is taken from a fit to the ISR and FNAL bubble chamber data (ref. [15]). The slopes of $R$ versus $A$ for the metallic targets are given by $0.129 \pm 0.004,0.129 \pm 0.004$, and $0.128 \pm 0.011$ at average energies of $160 \mathrm{GeV}, 260 \mathrm{GeV}$ and $520 \mathrm{GeV}$ respectively.

taken from the accelerator data [15]. The figure also includes data from four nuclear emulsion exposures at $200 \mathrm{GeV}$ [16-19] where the emulsions are considered as a composite target of effective $A=69^{\neq 3}$. A satisfactory fit to the data is given by

$\left\langle n_{\mathrm{c}}\right\rangle(\mathrm{p}-\mathrm{A})=\left\langle n_{\mathrm{c}}\right\rangle(\mathrm{p}-\mathrm{p}) A^{0.129 \pm 0.004}$

The ratio $R$ is independent of energy over the interval of $160 \lesssim 520 \mathrm{GeV}$ within statistics, although a slow increase with energy cannot be ruled out. The Monte

\#3 Since the values for $R$ from these four emulsion expts. were quite similar, the mean $R$ was computed and plotted in fig. 2a. The EFC model predicts $R=1+1 / 3\left(\vec{v}_{\mathrm{A}}-1\right)$ where $\bar{\nu}_{A}$ is the average number of nucleon collisions in the nucleus and was calculated from the formulation in ref. [4]. The CP model (6) predicts $R=(1 / 2)\left[1+A^{1 / 3} / 2\right]$. Our $\left(n_{\mathrm{C}}\right)$ is the $\bar{n}_{\mathrm{S}}$ of these emulsion experiments.
Carlo models alone give $R \approx A^{0.16}$ and $R \approx A^{0.11}$ lending further confidence to their utilization.

Angular distributions for the four heavier elements have been compared with those for carbon and hydrogen, using the variable $\eta_{\mathrm{p}}=\log \tan \theta_{\mathrm{p}}$. As spark chamber inefficiencies were important primarily at large angles, the observed (raw) multiplicity was taken equal to the true multiplicity at small angles. (At $160 \mathrm{GeV}, 90^{\circ}$ in p-p c.m. corresponds to $\sim 4.5^{\circ}$ in the laboratory and $\eta_{\mathrm{p}}$ of -1.12 for relativistic secondaries). In fig. $2 \mathrm{~b}-\mathrm{d}$, average multiplicities are plotted versus $A$ in three angular intervals. Observed average multiplicities are plotted in fig. $2 b$ for $\eta_{p} \leqslant-2.2$ (very forward prongs), and in fig. $2 \mathrm{c}$ for $\eta_{\mathrm{p}} \leqslant-1.12$. As a best measure of the average multiplicities in the backward hemisphere, these observed $\left\langle n_{\mathrm{c}}\right\rangle$ for $\eta_{\mathrm{p}} \leqslant-1.12$ were subtracted from the corrected total multiplicities, and these derived backward average multiplicities are plotted in fig. $2 d$. From these graphs, it is seen that 


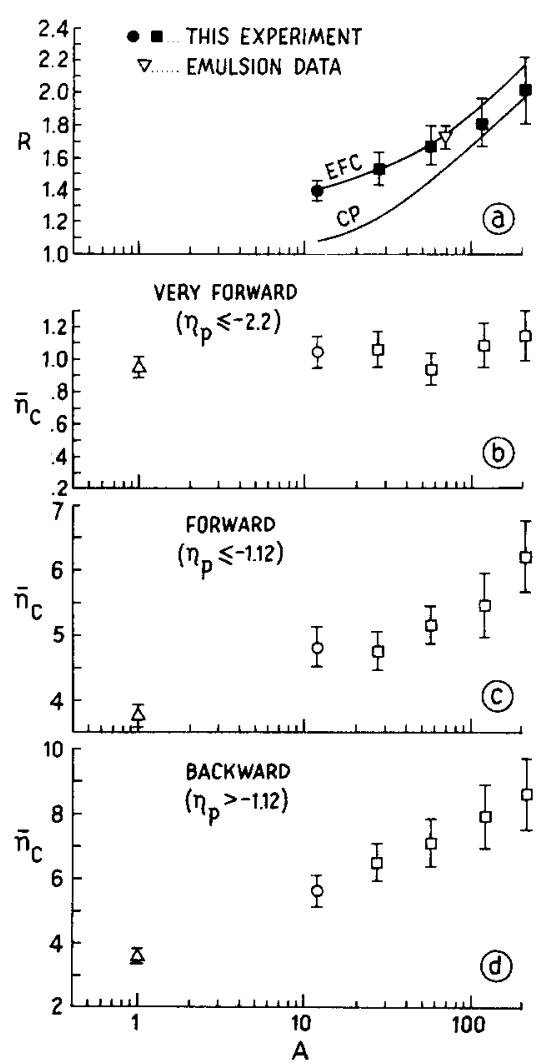

Fig. 2(a) The ratio $R$ of average charged multiplicities in heavy elements (table 1) to those in hydrogen versus mass number $A$. The emulsion point is the mean value of results from refs. [16-19]. The two lines are theoretical predictions from the Energy Flux Cascade Model of Gottfried [4] and the Coherent Production Model of Fishbane and Trefil [6]. The carbon point is represented by a separate symbol as that data was analyzed somewhat differently. The average energy is $160 \mathrm{GeV}$, and only statistical errors are shown. (b) Observed charged prong average multiplicities for very small angles, $\eta_{\mathrm{p}} \leqslant-2.2$, versus $A$ for the $160 \mathrm{GeV}$ data. (c) Observed charged prong average multiplicities for the "forward hemisphere", $\eta_{\mathrm{p}} \leqslant-1.12$, versus $A$ for the $160 \mathrm{GeV}$ data. (d) Charged prong average multiplicities for the "backward hemisphere", $\eta_{\mathfrak{p}}>-1.12$, versus $A$ for the $160 \mathrm{GeV}$ data (see text).

$\left\langle n_{\mathrm{c}}\right\rangle$ in the very forward direction is nearly constant and close to unity, indicating a "leading particle" in all targets. From fig. $2 \mathrm{c}$ and $2 \mathrm{~d} R=A^{0.084 \pm 0.006}$ for the forward hemisphere and $R=A^{0.167 \pm 0.008}$ for the backward hemisphere. From these data we conclude that the primary increase in multiplicity with $A$ comes from particles produced at larger angles.
The most obvious theoretical models, e.g., wherein each secondary may independently initiate a secondary cascade, predict much too rapid a rise in $\left\langle n_{\mathrm{c}}\right\rangle$ with $A$. A coherent production model gives closer agreement with the data [6], however, p-p bubble chamber data suggest that only a minority, $\sim 20 \%$ of the inelastic interaction, proceeds through this (diffraction or fragmentation) channel $[20,21]$. The best fit seems to be to the Energy Flux Cascade model of Gottfried [4] , as indicated in fig. 2a. Caution should be exercised in comparing data with these theoretical models as various authors differ in their formulation of $\bar{\nu}_{\mathrm{A}}$, the average number of nucleon interactions with a nucleus, as a function of $A$. Varying $\bar{\nu}_{\mathrm{A}}$ within reasonable limits can alter the model predictions as much as more fundamental changes in the models.

The first systematic attempt to study the variation of inclusive parameters of strong interactions with nuclear size at high energies confirms the remarkably slow increase in $\left\langle n_{\mathrm{c}}\right\rangle$ with $A$ as suggested by earlier cosmic ray data $[22-25]$. We believe that more detailed accelerator experiments of this type will shed further light on the early-time development of the final state of the $p-p$ interaction.

This experiment was a later stage of the Echo Lake Cosmic Ray Research Program of the University of Michigan and the University of Wisconsin. Among many valuable contributors to this experimental program were K.N. Erickson, G.D. DeMeester, B.W. Loo, F.E. Mills, Bruce Cork, P.V. Ramana Murthy, S. Lal, P.D. Kearney, R.F. Roth, D.E. Pellett, R. Hartung, S. Mikamo and D.H. Buress. Important technical assistance was provided by O. Haas, J. Pluta, J. Starkey, R. Brown, W. Winter, R. Beck, J. Hicks and the staff of the University of Wisconsin Physical Sciences Laboratory, Scanning and measuring assistance was provided by M. Caplan, F. Caplan, R. Haines, R. Smith and D. Twitty. We would also like to thank Professor Mario Iona and the other staff of the University of Denver for their cooperation in our use of the Echo Lake Laboratory.

\section{References}

[1] P.R. Vishwanath et al., Proc. 13th Intern. Conf. on Cosmic rays, University of Denver, Vol. 3, p. 2186 (1973). 
P.R. Vishwanath, doctoral thesis, University of Michigan (1974) (unpublished) UM HE 74-34.

[2] R.J. Wilkes, doctoral thesis, University of Wisconsin (1974) (unpublished).

[3] K.N. Erickson, doctoral thesis, Colorado State University, (1970) (unpublished); University of Michigan Report, UM HE 70-4 (unpublished); L.W. Jones et al. Acta Physica Academiae Scientiarum Hungaricae 29, Suppl. 3 (1970) 205.

[4] K. Gottfried, CERN preprint TH-1735 (1973); Phys. Rev. Letters 32 (1974) 957.

[5] A. Dar and J. Vary, Phys. Rev. D6 (1972) 2412.

[6] P.M. Fishbane and J.S. Trefil, Phys. Rev. Letters 31 (1973) 734

[7] A. Subramanian, Tata Institute of Fundamental Research, TIFR-BC-72-12 (1972).

[8] L.W. Jones, Proc. Intem. Conf. on Expectations for particle reactions at the new accelerators, University of Wisconsin, Madison, Wisconsin (1970).

[9] A.S. Goldhaber, Phys. Rev. D7 (1973) 765.

[10] L. Van Hove, CERN preprint TH-1746 (1973).

[11] S. Miyake, Proc. 13 th Intern. Conf. on Cosmic rays, University of Denver, 5 (1973) 3683.
[12] P.V. Ramana Murthy, Proc. 13th Intern. Conf. on Cosmic rays, University of Denver 5 (1973) 3668.

[13] N.A. Porter, Proc. 13th Intern. Conf. on Cosmic rays, University of Denver 5 (1973) 3656.

[14] L.W. Jones et al., Nucl. Phys. B43 (1972) 477.

[15] M. Antinucci et al., Nuovo Cimento Letters 6 (1973) 121.

[16] J. Babecki et al., Phys. Lett. 47B (1973) 268.

[17] J. Hebert et al., Phys. Letters 48B (1974) 467.

[18] J.R. Florian et al., Particles and Fields - 1973, 490, American Institute of Physics (1973).

[19] A. Gurtu et al., Phys. Lett. 50B (1974) 391.

[20] J.W. Chapman et al., Phys. Rev. Letters 32 (1974) 257.

[21] D.R.O. Morrison, CERN preprint D.Ph. II/PHYS 74-8 (1974).

[22] K. Rybicki and W. Wolter, Acta. Phys. Acad. Scient. Hung. 29, Suppl. 3 (1970) 143.

[23] S. Lal et al., Proc. 8th Intern. Conf. on Cosmic rays, Jaipur 5 (1963) 377.

[24] M. Miesowicz, Progress in elementary particle and cosmic ray physics, Vol. X (1971) 103.

[25] E.L. Feinberg, Phys. Rep. 5C (1972) 237. 